\title{
Gait training facilitates push-off and improves gait symmetry in children with cerebral palsy
}

Jakob Lorentzen ${ }^{1,2}$ Rasmus Frisk $^{1,2}$, Maria Willerslev-Olsen ${ }^{1,2}$, Laurent Bouyer,3 Simon Francis Farmer, ${ }^{4,5}$, and Jens Bo Nielsen ${ }^{1}$

1) Department of Neuroscience, Univ. of Copenhagen, Copenhagen, Denmark.

2) Helene Elsass Center, Charlottenlund, Denmark.

3) Department of Rehabilitation, University Laval, Quebec, Canada

4) Department of Clinical and Movement Neurosciences, Institute of Neurology of Neurology, University College London

5) Department of Clinical Neurology, National Hospital for Neurology and Neurosurgery, United Kingdom

Word Count: 4302

Keywords: Neuroplasticity; Cerebral palsy; Gait training

\section{Proof and correspondence to:}

Maria Willerslev-Olsen

Department of Neuroscience

University of Copenhagen,

Panum Institute 33.3

Blegdamsvej 3, 2200 Copenhagen N, Denmark.

E-mail: mwo@elsassfonden.dk 


\section{Abstract}

Background: Human walking involves a rapid and powerful contraction of ankle plantar flexors during pushoff in late stance $(\mathrm{dPF})$.

Objective: Here we investigated whether impaired dPF contributes to gait problems in children with cerebral palsy $(\mathrm{CP})$ and whether it may be improved by intensive gait training.

Methods: Sixteen children with CP (6-15 years) and fourteen typically developing (TD) children (4-15 years) were recruited. Foot pressure was measured by insoles and gait kinematics were recorded by 3-dimensional video analysis during treadmill and overground walking. Maximal voluntary plantar flexion (MVC) was measured while seated. Measurements were performed before and after a control period and after 4 weeks of 30 minutes daily inclined treadmill training.

Results: dPF and MVC were significantly lower in children with CP on the most affected (MA) as compared to TD children $(p<0.001)$. dPF was lower on the MA leg as compared to the less affected (LA) leg in children with CP $(p<0.05)$. Following gait training, increases in $\operatorname{dPF}(p<0.001)$ and MVC $(p<0.01)$ were observed for the MA leg. Following gait training children with CP showed similar timing of dPF and similar stance phase duration on both legs indicating improved symmetry of gait. These effects were also shown during overground walking.

Conclusion: Impaired ability to voluntarily activate ankle plantar flexors and produce a rapid and powerful push-off during late stance are of importance for impaired gait function in children with $\mathrm{CP}$. Intensive treadmill training may facilitate the central drive to ankle plantar flexors and reduce gait asymmetry during both treadmill and overground walking.

\section{Introduction}


Ankle plantar flexor muscles are of fundamental importance for human gait since they exert their action directly on the supporting ground and are in a position to both support the body against gravity and help to propel the body forward by providing a forward thrust at push-off ${ }^{1}$.

Several studies have demonstrated a relation between weakness of plantar flexors, reduced push-off force and gait speed in people with central motor lesions caused by stroke or cerebral palsy (CP) ${ }^{2-6}$. Impaired pushoff force will result in a) lack of stability during roll-off, b) impaired vertical and forward components of propulsion and c) impairment of leg flexion resulting in poor energy transfer from the swing leg to the trunk. Pathological gait asymmetry is likely to further impair these mechanisms. Such findings support the idea that the main functional challenge in patient populations with lesion of central motor pathways relates to muscle weakness due to lack of central drive and that a rational therapy should be directed towards strengthening muscle and improving the central drive to motoneurons ${ }^{7-12}$. Training that facilitates the power of ankle plantar flexors has indeed been shown to improve gait function in these patient populations ${ }^{7,13-16}$.

An additional problem for people with hemiplegic stroke and cerebral palsy (either childhood hemiplegia or asymmetric diplegia or quadriplegia) is that asymmetry of gait is suspected to result in long-term trauma to joints, tendons and muscles and thus may increase the risk of arthritis ${ }^{17}$. Impaired push-off force and forward propulsion on the most affected side has been shown to be a major determinant of asymmetry of gait in individuals with hemiplegic stroke ${ }^{18-20}$ and therapy directed towards improving push-off force may therefore also help to adjust asymmetries of gait and prevent possible long-term mechanical deterioration ${ }^{21}$. The recent initiation of central registration of biomechanical and clinical data in children and adults with CP in the Scandinavian countries promises to provide important information about such long-term effects ${ }^{22}$.

The present study is based on a re-analysis of a previous gait training study designed to facilitate dorsiflexor force and toe lift in the swing phase of gait in children with $\mathrm{CP}{ }^{23,24}$. Children were asked to train at home for 30 minutes daily for 4 weeks on a treadmill with progressive incline and progressive speed. Here we hypothesized that the training would not only improve function of dorsiflexors, but also facilitate activation of plantar flexors in stance to produce increase of the maximum rate of change of push off force. We further 
hypothesized that the training would reduce gait asymmetry and that these effects on gait function would be transferrable to overgound walking.

\section{Methods}

\section{Participants}

Sixteen children with cerebral palsy (CP) (Table 1; mean age 9.4 years, range 4-13; 11 boys, 5 girls) were recruited through the Danish Cerebral Palsy Organisation. Neurological evaluation was performed by an experienced physician (JBN) and therapist (JL). All children had impaired gait function with some degree of foot drop and/or toe walking. All children were classified as spastic CP with unilateral or bilateral weakness of the lower limbs. 12 were classified as hemiplegic ( 8 right sided and 4 left sided) and 4 as diplegic. All children showed greater or lesser asymmetry of gait with a most affected (MA) and less affected (LA) side. None of the children had received any corrective surgery of the lower limbs. 8 children had received Botulinum toxin treatment, but not within the past 6 months. The severity of CP was classified according to the Gross Motor Classification System (GMFCS ${ }^{25}$ ). The clinical characteristics of the children are given in Table I.

The study was approved by the local ethics committee (H-B-2009-017) and all procedures were conducted within the standards of the Helsinki declaration. Prior to the experiments parents and children received written and verbal information. Written consent for participation was obtained.

Kinematic measures were obtained in the children with CP at four different experimental sessions (twice before and twice after training with one-month interval between each measurement).

14 typically developing (TD) children were recruited for the study (age 10.2 years, range 4-15; 8 boys, 6 girls) in order to provide normative data. TD children were only tested once and thus did not participate in any training. 


\section{Experimental set up}

Gait function was evaluated in the children with CP during four separate test sessions at intervals of approximately one month. All test sessions took place at the Helene Elsass Center (www.elsasscenter.dk; Charlottenlund, Denmark). The test sessions were carried out at recruitment (1pre) and then again after a one-month control period (2pre) in order to assess the reproducibility of measurements. Gait training was initiated the day after the second control test session. The last two test sessions took place the last day of training (1post) and again one month after end of training (2post).

\section{Gait analysis during treadmill and over ground walking}

The gait of the children was analysed by recording the leg movements using a 3-D motion capture system. The children were asked to walk on a treadmill as well as over ground. Measurements during treadmill training were obtained since the training took place at a treadmill and in order to compare kinematics at the same gait speed before and after training during controlled conditions at the treadmill. Following 5 minutes of familiarisation to treadmill walking in the beginning of the first test session, the children selected their own comfortable walking speed. It varied between $2 \mathrm{~km} / \mathrm{h}$ and $4 \mathrm{~km} / \mathrm{h}$ with a mean of $2.8 \mathrm{~km} / \mathrm{h} \pm 0.6$. This selfchosen walking speed was used for measurements in all subsequent test sessions during treadmill walking. All the children were asked to hold on to the bars of the treadmill for support.

Measurements during over ground walking were obtained in order to investigate whether findings obtained during treadmill walking could be translated into over ground walking. For over ground walking children were asked to walk back and forth in a corridor where a five-meter lane had been marked. Children were asked to walk at their preferred walking speed at all sessions. Two-minute recordings were obtained for plantar pressure analysis. 3D motion capture was not feasible during over ground walking.

\section{Motion analysis}


Kinematic data were captured using a Qualysis motion capture system (Qualisys, Gothenburg, Sweden) with six, synchronous Oqus 1 cameras operating at a sampling frequency of $200 \mathrm{~Hz}$. Reflective markers (Size $12 \mathrm{~mm}$ ) were placed bilaterally at 1) the hip, 2) the lateral articular line of the knees, 3) the lateral malleolus and 4) the lateral side of the $5^{\text {th }}$ metatarsal.

\section{Plantar pressure measurement}

Pressure sensitive soles were placed in the children's shoes in order to record the plantar pressure from each foot during walking (F-scan wireless, Tekscan, CA Mätsystem, Sweden). The pressure sensitive soles were fitted individually for the shoes of each child and reused by that child for each of the four test sessions. Prior to each recording, the system was calibrated by having the child standing relaxed while holding on to the rails of the treadmill with equal weight on the two legs. This measurement was not possible for technical reasons in 5 of the children with $\mathrm{CP}$ at either pre-test 2, post-test 1 or post-test 2 and data from these children was therefore omitted when comparing data across the different sessions.

\section{Measurement of maximal voluntary contraction strength (MVC)}

In order to measure MVC, children were placed in a dynamometer (custom-build, Thomas Sinkjær \& Jakob Buus Andersen, University of Aalborg, Denmark), which allowed isometric contractions of ankle muscles against a foot plate at fixed hip (100 deg.), knee (90 deg.) and ankle joint positions (100 deg.). This position was chosen to ensure comfortable support for the children and relatively selective activation of the soleus muscle during MVC. This was checked by meausring EMG activity from two sets of bipolar electrodes (Ambu Blue sensor N,N-10-A/25. Ambu A/S Ballerup, Recording area $0.5 \mathrm{~cm}^{2}$ inter-electrode distance, $2 \mathrm{~cm}$ ) placed at the proximal end of the Tibialis anterior (TA) muscle and over the soleus muscle below the gastrocnemii muscles. The skin was brushed softly with sandpaper (3M red dot; 3M, Glostrup, Denmark). EMG signals were sampled by a wireless EMG recording system (BTSFreeEMG; BTS Bioengineering Corp.Brooklyn, NY, USA). Data was filtered (band-pass, $10 \mathrm{~Hz}-400 \mathrm{~Hz}$ ), sampled at $1000 \mathrm{~Hz}$, and stored on a PC for off-line analysis. 
Children were verbally encouraged to perform a plantar flexion as quickly and strongly as possible. Children were carefully explained how to perform the contraction and were allowed several attempts to get familiarized with the setup before performing the actual measurements. Consistency was ensured once measures were obtained with little variability between the two baseline sessions. None of the children showed TA EMG activity during the MVC tests. Activation of knee muscles was not checked by EMG recording, but by observation and palpation of the tension of the muscle bellies.

Three trials were performed at intervals of 20-30 s. The trial with the largest torque measured from the foot plate was selected. It was difficult for some of the children to understand how to perform the task and exert maximal effort. It was therefore only possible to use MVC data from 10 of the children. MVC measurements were not obtained in TD children.

In the same experimental session and with the leg in the same position, supramaximal electrical stimulation was applied to the tibial nerve at the popliteal fossa (Stimulator: Digitimer, DS4, Cambridge, UK) in order to determine the strength of the muscle independently of voluntary neural drive. The cathode was a ball electrode placed over the nerve, whereas the anode was a plate placed above the patella. The stimulus was a rectangular pulse with a duration of $1 \mathrm{msec}$. Single stimuli were applied at intervals of $10 \mathrm{~s}$ while stimulation intensity was increased until the recorded force from the foot plate and the maximal M-response recorded in the soleus EMG no longer increased with increasing stimulus intensity. The maximal torque elicited was determined (Tmax). 5-10 stimuli were usually necessary to determine Tmax in each child. Seven of the children with $\mathrm{CP}$ (or their parents) did not tolerate maximal electrical stimulation and data were therefore obtained from 9 children with CP. MVC was also obtained in all these children. Tmax measurements were not obtained in TD children.

\section{Training protocol}


Only children with CP performed treadmill training. Training sessions were scheduled for 30 minutes every day for four consecutive weeks at home. All participants had a treadmill (ECO II G6432N) delivered to their home several days prior to initiating the training. The children could split up the 30 minutes of walking in multiple periods of shorter duration as long as 30 minutes of total walking time was achieved by the end of the day.

The speed and incline settings of the treadmill for the initial training was based on the settings for the first test session. As the child's gait improved and they became more comfortable with incline and speed, the settings could be increased. Parents and children were instructed to increase primarily the treadmill incline. The children were also instructed to make an effort in placing the heel on the treadmill in early stance. The parents were instructed to encourage the child as much as possible both with respect to maintenance and progression of the training and with the placement of the heel in early stance. Attention was thus focussed on walking on an incline and on facilitating activity of the dorsiflexors in a fast but controlled pace without running. All children wore shoes without any inserts or orthosis during the testing. During the training period children were instructed to walk barefoot or with shoes without any inserts.

All children and their parents were handed a diary in order to record how long time the child had been training each day, the speed of the treadmill, the incline of the treadmill and what other activities they had been participating in during the day.

The children on average walked 25.7 minutes (Range: 14.4-30, SD: 4.8) daily on the treadmill. They progressively increased their walking speed from $2.4 \mathrm{~km} / \mathrm{h}$ to $3.3 \mathrm{~km} / \mathrm{h}$ (Range: $2-5.9 \mathrm{~km} / \mathrm{h}, \mathrm{SD}: 0.55$, SD: 1.16 ), corresponding to an increase of $19 \%$ and the incline on the treadmill from $7.5 \%$ to $10 \%$ (Range: $5-12 \%$ SD: 2.3, SD: 2.76), corresponding to an increase of $25 \%$. All children found the training strenuous in the beginning, but all of them, nonetheless completed the full training period and reported that the training was less hard towards the end of the training period. 


\section{Off-line data analysis}

Signal processing and analysis was carried out off line. All motion and plantar pressure data were imported into Matlab (Mathworks, Massachusetts, USA) for further analysis.

\section{Measurement of rate of change of push-off force during gait}

Movement of the ankle joint was calculated from the position of the reflective markers throughout the gait cycle for both legs (Fig. 1A, C). The push-off velocity was calculated by differentiating the movement of the ankle joint in the second part of the stance phase and identifying the peak of the derivative (Fig. $1 \mathrm{~A}, \mathrm{C}$ ). Pressure on the forefoot was calculated at different times during the gait cycle by averaging all measuring points covering the most distal $1 / 3$ of the foot (Fig. 1B, D). The pressure at push-off was quantified by differentiating this force measurement and identifying the peak of the derivative (i.e. peak rate of change of force; Fig. 1B, D). We refer to this as the peak derivative of ground reaction force at push off (dPF), while realizing that the force measured is only the vertical component of the actual force applied to the ground, rather than a force applied in the direction of the movement of the body. To take differences in body weight among the children into account dPF was normalized by relating the peak of the derivative to the peak force. This was done separately for each leg. All measures were averaged over all steps within the recording period.

\section{Calculation of symmetry indexes}

In order to compare symmetry of gait for TD children and children with $\mathrm{CP}$ as well as before and after gait training for children with $\mathrm{CP}$, symmetry indexes were calculated by dividing measurements on the MA and LA leg in children with CP and the left and right leg in TD children. Symmetry indexes were obtained for 1) the stance phase duration (calculated from the interval between ground contact and toes off for each leg. 2) The amplitude of the dPF. 3) The timing of dPF on the two sides with respect to each other. In order to calculate the latter index, we first determined the time of the maximal dPF for each leg in each step. This 
resulted in two time series which were then analysed using simple cross-correlation. This resulted in crosscorrelation peaks located at equal distance before and after time zero; i.e. the right leg preceded and followed the left leg with a similar time. The asymmetry index was therefore calculated as the relation between the time from zero to the two cross-correlation peaks.

\section{Statistics}

Assumptions of normality of the distribution for all variables were explored through histograms and normality plots and confirmed with the Shapiro-Wilk's normality test. One Way RM ANOVA was used to determine differences in measurements at the four test sessions in MVC, dPF and over-ground gait speed. Student-Newman-Keuls (SNK) postHoc test was used to determine significant differences between individual test sessions. If nothing else is stated correlation coefficients $\left(r^{2}\right)$ are given for regression lines calculated for the relation between different measures. Pearson's bivariate correlation analysis was used to explore the statistical significance of associations between the different measures. 1-way ANCOVA was used to test for differences in the relation between $\mathrm{DPF}$ and gait speed between the LA and MA leg in children with CP and TD children. Differences in average values between the populations was tested using SNK posthoc test. All analyses were performed with Sigmaplot 12.5 (SYSTAT Software, San Jose, CA, USA) for Windows.

\section{Results}

\section{Comparison between children with CP and TD children.}

Treadmill and overground gait speed were correlated to the peak rate of change in push-off force exerted by the forefoot ( $\mathrm{dPF}$ for both the MA (Fig. 2A-B; closed circles) and LA leg of children with CP (Fig. 2A-B; open circles) as well as for TD children (Fig. 2A-B; open triangles) with correlation coefficients varying between 0.42 and 0.77 . A significantly steeper gradient in the relationship between dPF and gait speed was found for the MA leg in children with CP than for TD children for both treadmill gait (ANCOVA; $F=5.07 ; p=0.03$ ) and 
overground gait (ANCOVA; $F=5.07 ; p=0.03$ ). No difference in slope gradient was found between the LA leg in children with CP and TD children. Posthoc tests showed significantly higher dPF for the LA leg in children with $\mathrm{CP}$ and in TD children than for the MA leg in children with CP (SNK; $p<0.01)$.

Neither treadmill (Fig. 2C) nor overground dPF (Fig. 2E) were significantly correlated to MVCpf for the MA leg in children with $\mathrm{CP}$, although an almost significant relation was found for the treadmill $\mathrm{dPF}\left(\mathrm{r}^{2}=0.33\right.$; $\mathrm{p}=0.056$ ). There was also no significant correlation between treadmill and overground gait speed and MVCpf (Fig. 2D \& F).

\section{Effect of gait training on push-off ground reaction force.}

As already described dPF was lower on the MA side than on the LA side prior to training (Fig. 3A-B; data from the LA leg are shown as shaded grey rectangle). Training had no effect on the LA leg, but improved dPF significantly for the MA leg. Following training the difference in dPF between the two legs consequently diminished and the children had a similar dPF for both legs during both treadmill (Fig. 3A) and overground (Fig. 3B) walking (Fig. 3A-B; compare columns 3-4 to shaded grey rectangle). One way RM ANOVA thus revealed a significant effect of test session $(F=6.5, p<0.001$ for treadmill walking and $F=8.5 ; p<0.001$ for overground walking) and posthoc test (SNK) revealed a significance increase at both sessions following training compared to the two sessions prior to training $(p<0.05)$ for both treadmill and overground walking. There were no significant changes in the size of the torque evoked by supramaximal stimulation of the tibial nerve following training, signifying that the training was not accompanied by increased contractile force of the plantar flexor muscles (Fig. 3C; $F=0.01 ; p=0.9$ ). The maximal voluntary plantar flexion (MVCpf), in contrast, showed significant interaction with session (Fig. 3D; $F=8.6 ; \mathrm{p}<0.001$ ) and posthoc tests demonstrated significant increase at both post training sessions with respect to the first pretraining session $(p<0.001)$, but not the second pretraining session $(p=0.13$ and $p=0.3$ respectively for the first and second post training sessions). There were no significant differences in MVCpf between the two sessions prior to training 
(Fig. 3D; $p=0.18$ ). When calculating the percentage change in $\mathrm{PPF}$ and MVCpf from before (pre-test 1 ) to after training (post-test 1), a significant correlation was found between the change in MVCpf and dPF during both treadmill and overground gait (Fig. 3E-F; Treadmill: $r^{2}=0.52 ; p<0.001 ;$ overground: $r^{2}=0.24 ; p<0.05$ ), indicating that the children who increased their MVCpf were the same children who increased dPF in the MA leg.

\section{Effect of gait training on symmetry of gait}

In order to quantify side to side differences in the gait of the children with CP we divided the duration of the stance phase and magnitude of dPF during treadmill and overground walking on the MA side by the corresponding measurements taken from the LA side. Stance phase ratio values $>1$ indicate MA/LA asymmetry; dPF ratio values $<1$ indicate MA/LA push-off force asymmetry. We quantified the cadence of gait further by recording the timing of the MA and LA dPFs and expressing this as a ratio where values $>1$ indicate gait timing asymmetry. Fig. 4 shows these relations calculated for the $1^{\text {st }}$ session prior to training (black columns) and the first session immediately after the training (grey columns). A significant change in the relation between the two sides towards a more symmetric gait was observed for both the duration of the stance phase $(p<0.01)$, overground $\operatorname{dPF}(p<0.01)$, timing ratios of the treadmill and overground $\operatorname{dPF}(p<0.001)$. The change observed for treadmill dPF ratio whilst tending towards improved symmetry just failed to reach statistical significance ( $p=0.064$, see Fig. 4 , second column). The range of symmetry indexes calculated for TD children is indicated as a shaded grey rectangle in the graph for comparison.

\section{Age-related difference in training effect}

Not all children responded to the training. When plotting the increase in MVCpf following the training as a function of age a significant negative correlation was found (Fig. $5 A ; r^{2}=0.56 ; p=0.003$ ). This was also the case when plotting the change in symmetry index for the timing of dPF during overground walking (Fig. 5B; $\left.r^{2}=0.56 ; p=0.007\right)$. A negative correlation with age was also found for the magnitude of dPF during treadmill 
gait (Fig. 5C; $r^{2}=0.27 ; p=0.1$ ) and during overground walking (Fig. 5D; $r^{2}=0.25 ; p=0.051$ ), but none of these correlations were significant.

\section{Discussion}

This study has demonstrated that reduced ability to produce voluntary plantar flexion force is correlated with reduced peak rate of change of push-off force (dPF) during treadmill gait in children with CP. It has further been shown that gait training may increase the voluntary plantar flexion torque and push-off force in the legs of children with $\mathrm{CP}$. The training related improvements in these parameters result in a significantly more symmetric gait in children with CP. This effect on symmetry of gait was shown to transfer to overground walking. We suggest that impaired plantar flexion effort resulting in reduced dPF should be an important therapeutic target in children with CP.

\section{Increase in MVC with training}

It is surprising that maximal voluntary plantar flexion effort increased significantly in the children with CP following gait training, since such large increases are normally associated with specific strength training programmes ${ }^{26-28}$. However, the gain in MVC should be seen in relation to the very low voluntary muscle strength in the children prior to the training at the two baseline sessions. Importantly, there were no changes in the strength of the muscle itself as determined from the maximal torque elicited by supramaximal nerve stimulation (Tmax). This indicates that the increase in MVC was related to increased central neural drive to the plantar flexor motor neuron pools rather than peripheral changes in the muscle contraction properties. Our data show that daily treadmill training has helped to optimize central neural drive to the ankle plantar flexor muscles, which transfers to both increased MVC and dPF and more symmetric overground walking.

\section{What determines gait ability?}


The present findings confirm the results of a large number of studies in the past two decades, which have indicated that reduced muscle strength is the main functional problem in people with central motor lesion such as stroke and $\mathrm{CP}$, whereas spasticity - at least in the sense of hyperexcitable reflex activity - is of less or no importance ${ }^{7-12} 2,11,29-36$. Similar to what has been reported also in other studies we found that voluntary plantar flexor muscle strength was related to dPF and self-chosen gait speed on the treadmill and overground prior to training ${ }^{13}$. Following training the increase in MVC was correlated to improved dPF as well as improved symmetry of walking during both treadmill and overground walking. This indicates that stronger central neural activation of the plantar flexor muscles is a key factor determining the improvement of gait speed.

\section{Age-related training effects}

We found that only children younger than 10-12 years showed increased MVCpf and increased dPF following the training (Fig. 5). Willerslev-Olsen et al. (2015) studying the same cohort of children with CP found that increased beta and gamma band coherence between populations of Tibialis anterior motor units following gait training was restricted to children younger than 10-12 years of age. They argued that the increased coherence in young children was best explained by plastic changes in central motor pathways leading to increased central drive to ankle dorsiflexors. In older children (>10-12 years) the treadmill training programme appeared insufficient to produce similar plastic changes. We suggest that compared to older children and adults, children younger than 10-12 years are in a more developmentally sensitive period where exercise induced plastic changes are more easily induced in the central motor pathways that deliver the central drive involved in the control of gait. It is important to stress, however, that our findings do not indicate that older children and adults cannot benefit from gait training. They may benefit in different ways (i.e. increased movement range, longer endurance etc). The plastic changes found here for the younger children 
may also take longer to induce in older children and adults and thus require more than the 4 weeks of training that we investigated.

\section{Symmetry of gait}

It has been shown in a number of studies that normal gait is seldom fully symmetric and that step length and push-off force may vary between the two legs in neurologically-intact individuals without any apparent link to leg dominance ${ }^{37,38}$. However, this variation in step length, push-off force and other gait parameters is much smaller than that seen in hemiplegic stroke survivors and individuals with hemiplegic $\mathrm{CP} 18,20,39$. It is generally believed that the asymmetry of gait in people with hemiplegia is a problem that requires consideration in its own right because of the risk of inappropriate loading of joints, muscles and tendons with consequent pain and osteoarthritis ${ }^{17}$. The observation that the treadmill training improved dPF and gait timing on the MA side in the children with CP during both treadmill and overground walking leading to a significant reduction in gait asymmetry should be seen as a beneficial effect of the training in its own right. It indicates that training induced reduction of gait asymmetry has the potential to reduce long term tear and load on more proximal joints, muscles and tendons, which may translate to a reduction in long-term pain and osteoarthritis. Long-term follow up studies will be required in order to answer this question.

\section{Limitations of the study}

It should be pointed out that we deliberately decided not to use a force platform to measure ground reaction forces which is the conventional way of determining the push-off force during gait ${ }^{40}$. The reason for this is that we wished to compare directly the force measurements during treadmill and overground walking and to obtain an average measure over a larger number of steps. Furthermore, the derivative of the change of force quantifies the propulsive kick needed to launch the leg into swing. We argue therefore that the dPF measure during gait captures the explosive increase in central drive required for leg push off from stance $40,41$. 
It should also be pointed out that the demonstration of correlation between dPF and gait speed during both treadmill and overground walking does not tell us anything about the causal relationship between the two parameters. Gait speed may increase because of increased dPF or the other way around.

Finally, we did not measure MVC and Tmax on the LA leg in children with CP. We therefore cannot say whether the training also affected muscle strength on the LA leg. The lack of change in gait parameters on the LA leg following the training (Fig. 3) suggests that major changes in MVC on that side are unlikely.

\section{Conclusion}

We suggest that impaired ability to voluntarily activate ankle plantar flexors and produce a rapid and powerful push-off at end of stance is of importance for asymmetric gait in children with CP. We further argue that intensive gait training in children with CP may facilitate voluntary control of ankle plantar flexors and reduce gait asymmetry by increasing push-off force.

\section{Acknowledgement}

We are grateful to Michael Hedegård for taking care of the logistic of the project. We thank Dr Lucinda Carr for her critical reading of the manuscript. The study was supported by a grant from Ludvig and Sara Elsass Foundation. SFF acknowledges funding support from the National Institutes for Health Research University College London Hospitals Biomedical Research Centre. LB was supported in part by the Natural Sciences and Engineering Research Council of Canada. 



\section{References}

1. Winter DA. Energy generation and absorption at the ankle and knee during fast, natural, and slow cadences. Clinical orthopaedics and related research. 1983(175):147-154.

2. Nadeau S, Gravel D, Arsenault AB, Bourbonnais D. Plantarflexor weakness as a limiting factor of gait speed in stroke subjects and the compensating role of hip flexors. Clinical biomechanics. 1999;14(2):125-135.

3. Mueller MJ, Minor SD, Schaaf JA, Strube MJ, Sahrmann SA. Relationship of plantar-flexor peak torque and dorsiflexion range of motion to kinetic variables during walking. Physical therapy. 1995;75(8):684-693.

4. Olney SJ, MacPhail HE, Hedden DM, Boyce WF. Work and power in hemiplegic cerebral palsy gait. Physical therapy. 1990;70(7):431-438.

5. Eek MN, Beckung E. Walking ability is related to muscle strength in children with cerebral palsy. Gait \& posture. 2008;28(3):366-371.

6. Chen $\mathrm{G}$, Patten $\mathrm{C}$. Joint moment work during the stance-to-swing transition in hemiparetic subjects. Journal of biomechanics. 2008;41(4):877-883.

7. Pak S, Patten C. Strengthening to promote functional recovery poststroke: an evidence-based review. Topics in stroke rehabilitation. 2008;15(3):177-199.

8. Patten C, Lexell J, Brown HE. Weakness and strength training in persons with poststroke hemiplegia: rationale, method, and efficacy. Journal of rehabilitation research and development. 2004;41(3A):293-312.

9. Damiano DL, Quinlivan J, Owen BF, Shaffrey M, Abel MF. Spasticity versus strength in cerebral palsy: relationships among involuntary resistance, voluntary torque, and motor function. European journal of neurology. 2001;8 Suppl 5:40-49.

10. Moreau NG, Falvo MJ, Damiano DL. Rapid force generation is impaired in cerebral palsy and is related to decreased muscle size and functional mobility. Gait \& posture. 2012;35(1):154-158.

11. Geertsen SS, Kirk H, Lorentzen J, Jorsal M, Johansson CB, Nielsen JB. Impaired gait function in adults with cerebral palsy is associated with reduced rapid force generation and increased passive stiffness. Clinical neurophysiology : official journal of the International Federation of Clinical Neurophysiology. 2015;126(12):2320-2329.

12. Gillett JG, Lichtwark GA, Boyd RN, Barber LA. Functional capacity in adults with cerebral palsy: Lower limb muscle strength matters. Archives of physical medicine and rehabilitation. 2018.

13. Eek MN, Tranberg R, Zugner R, Alkema K, Beckung E. Muscle strength training to improve gait function in children with cerebral palsy. Developmental medicine and child neurology. 2008;50(10):759-764.

14. Kirk H, Geertsen SS, Lorentzen J, Krarup KB, Bandholm T, Nielsen JB. Explosive Resistance Training Increases Rate of Force Development in Ankle Dorsiflexors and Gait Function in Adults With Cerebral Palsy. Journal of strength and conditioning research. 2016;30(10):2749-2760.

15. Teixeira-Salmela LF, Olney SJ, Nadeau S, Brouwer B. Muscle strengthening and physical conditioning to reduce impairment and disability in chronic stroke survivors. Archives of physical medicine and rehabilitation. 1999;80(10):1211-1218.

16. Milot MH, Nadeau S, Gravel D, Bourbonnais D. Effect of increases in plantarflexor and hip flexor muscle strength on the levels of effort during gait in individuals with hemiparesis. Clinical biomechanics. 2008;23(4):415-423.

17. Murphy KP. Cerebral palsy lifetime care - four musculoskeletal conditions. Developmental medicine and child neurology. 2009;51 Suppl 4:30-37.

18. Allen JL, Kautz SA, Neptune RR. Forward propulsion asymmetry is indicative of changes in plantarflexor coordination during walking in individuals with post-stroke hemiparesis. Clinical biomechanics. 2014;29(7):780-786. 
19. Roerdink M, Beek PJ. Understanding inconsistent step-length asymmetries across hemiplegic stroke patients: impairments and compensatory gait. Neurorehabilitation and neural repair. 2011;25(3):253-258.

20. Lauziere S, Mieville C, Betschart M, Aissaoui R, Nadeau S. Plantarflexor weakness is a determinant of kinetic asymmetry during gait in post-stroke individuals walking with high levels of effort. Clinical biomechanics. 2015;30(9):946-952.

21. Lauziere S, Mieville C, Betschart M, Duclos C, Aissaoui R, Nadeau S. A more symmetrical gait after split-belt treadmill walking increases the effort in paretic plantar flexors in people post-stroke. Journal of rehabilitation medicine. 2016;48(7):576-582.

22. Hagglund G, Andersson S, Nordmark E, Sunden G, Westbom L. [All children with cerebral palsy are systematically followed-up. A project on cooperation between pediatric orthopedics and pediatric rehabilitation in Southern Sweden]. Lakartidningen. 1998;95(15):1666-1667.

23. Willerslev-Olsen M, Lorentzen J, Nielsen JB. Gait training reduces ankle joint stiffness and facilitates heel strike in children with Cerebral Palsy. NeuroRehabilitation. 2014;35(4):643-655.

24. Willerslev-Olsen M, Petersen TH, Farmer SF, Nielsen JB. Gait training facilitates central drive to ankle dorsiflexors in children with cerebral palsy. Brain : a journal of neurology. 2015;138(Pt 3):589603.

25. Palisano R, Rosenbaum P, Walter S, Russell D, Wood E, Galuppi B. Development and reliability of a system to classify gross motor function in children with cerebral palsy. Developmental medicine and child neurology. 1997;39(4):214-223.

26. Gillett JG, Boyd RN, Carty CP, Barber LA. The impact of strength training on skeletal muscle morphology and architecture in children and adolescents with spastic cerebral palsy: A systematic review. Research in developmental disabilities. 2016;56:183-196.

27. Ross SM, MacDonald M, Bigouette JP. Effects of strength training on mobility in adults with cerebral palsy: A systematic review. Disabil Health J. 2016;9(3):375-384.

28. Bania TA, Dodd KJ, Baker RJ, Graham HK, Taylor NF. The effects of progressive resistance training on daily physical activity in young people with cerebral palsy: a randomised controlled trial. Disability and rehabilitation. 2016;38(7):620-626.

29. Jonkers I, Delp S, Patten C. Capacity to increase walking speed is limited by impaired hip and ankle power generation in lower functioning persons post-stroke. Gait \& posture. 2009;29(1):129-137.

30. Williams G, Banky M, McKenzie D, Olver J. Ankle plantarflexor spasticity is not differentially disabling for those who are weak following traumatic brain injury. Brain injury. 2017;31(2):193-198.

31. Kremer TR, Van Dillen LR, Wagner JM. Dynamometer-based measure of spasticity confirms limited association between plantarflexor spasticity and walking function in persons with multiple sclerosis. Journal of rehabilitation research and development. 2014;51(6):975-984.

32. Wagner JM, Kremer TR, Van Dillen LR, Naismith RT. Plantarflexor weakness negatively impacts walking in persons with multiple sclerosis more than plantarflexor spasticity. Archives of physical medicine and rehabilitation. 2014;95(7):1358-1365.

33. You YY, Chung SH, Lee HJ. Impact of the difference in the plantar flexor strength of the ankle joint in the affected side among hemiplegic patients on the plantar pressure and walking asymmetry. $J$ Phys Ther Sci. 2016;28(11):3015-3019.

34. Lamontagne A, Malouin F, Richards CL. Contribution of passive stiffness to ankle plantarflexor moment during gait after stroke. Archives of physical medicine and rehabilitation. 2000;81(3):351358.

35. Lamontagne A, Richards CL, Malouin F. Coactivation during gait as an adaptive behavior after stroke. Journal of electromyography and kinesiology : official journal of the International Society of Electrophysiological Kinesiology. 2000;10(6):407-415.

36. Nadeau S, Arsenault AB, Gravel D, Bourbonnais D. Analysis of the clinical factors determining natural and maximal gait speeds in adults with a stroke. American journal of physical medicine \& rehabilitation. 1999;78(2):123-130. 
37. Kaczmarczyk K, Blazkiewicz M, Wit A, Wychowanski M. Assessing the asymmetry of free gait in healthy young subjects. Acta Bioeng Biomech. 2017;19(3):101-106.

38. Riskowski JL, Hagedorn TJ, Dufour AB, Hannan MT. Functional foot symmetry and its relation to lower extremity physical performance in older adults: the Framingham Foot Study. Journal of biomechanics. 2012;45(10):1796-1802.

39. Prosser LA, Lauer RT, VanSant AF, Barbe MF, Lee SC. Variability and symmetry of gait in early walkers with and without bilateral cerebral palsy. Gait \& posture. 2010;31(4):522-526.

40. Lipfert SW, Gunther M, Renjewski D, Seyfarth A. Impulsive ankle push-off powers leg swing in human walking. The Journal of experimental biology. 2014;217(Pt 8):1218-1228.

41. Aagaard P, Simonsen EB, Andersen JL, Magnusson P, Dyhre-Poulsen P. Increased rate of force development and neural drive of human skeletal muscle following resistance training. Journal of applied physiology. 2002;93(4):1318-1326. 

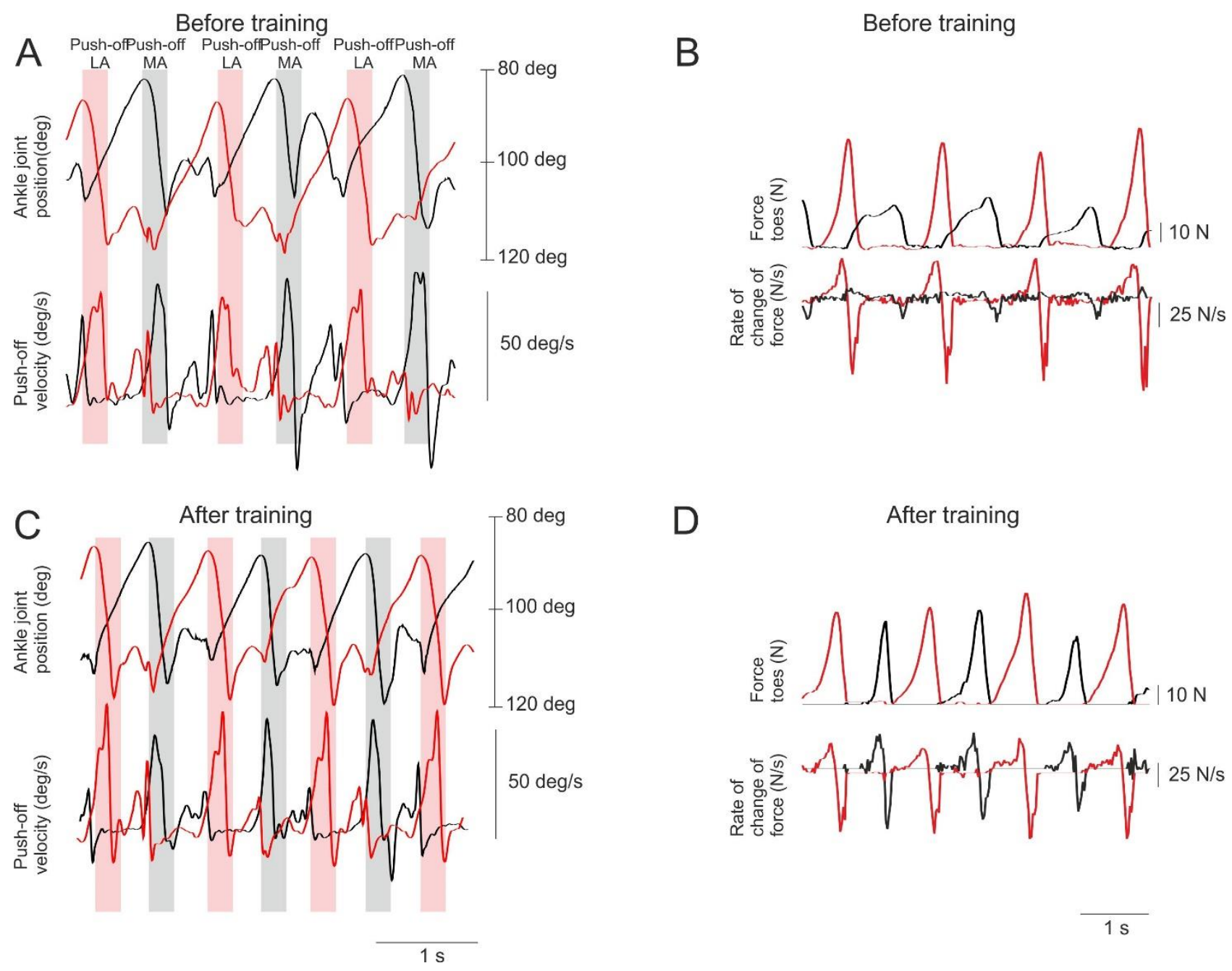

Figure 1. Ankle joint movement and push-off velocity $(A, C)$ and force $(B, D)$ in a single child with cerebral palsy before $(A-B)$ and after gait training (C-D).

Position of the ankle joint (upper traces in A \& C) was calculated from the relative position of the reflexive markers throughout the gait cycle for the least (red lines) and most (black lines) affected leg throughout the gait cycle. It is given in deg and scale bars are indicated to the right of the races. The velocity of the changes in ankle joint position was obtained by differentiating the ankle joint position measurements (lower traces in A \& C). The peak velocity was taken as the velocity at push-off. The time period towards the end of stance where the ankle joint moves rapidly in plantarflexion is indicated for each leg by shaded red and grey bars. The peak push-off velocity occurred within this period as can be seen. Data from three gait cycles are shown. The time is indicated by a horizontal scale bar to the lower right in C. Note, the larger push-off velocity following training for the MA leg.

Force was measured from a foot sole placed under each foot of the child which allowed a detailed map of the force distribution for each time point throughout the gait cycle. The force corresponding to the forefoot ( $B$ \& $D$, upper traces) was calculated by setting an area corresponding to the distal most $1 / 3$ of the foot sole and averaging the force for each timepoint throughout the gait cycle. Data from the MA leg are shown in black and data from the LA leg in red. The velocity of force changes ( $B \& D$, lower traces) was calculated by differentiating the force measurements and dPF was determined by identifying the peak of the velocity of force changes and normalising for the peak force. Data from 4 gait cycles are shown. The time is indicated by a horizontal scale bar to the lower right in D. Note, the larger push-off force following training for the MA leg. 

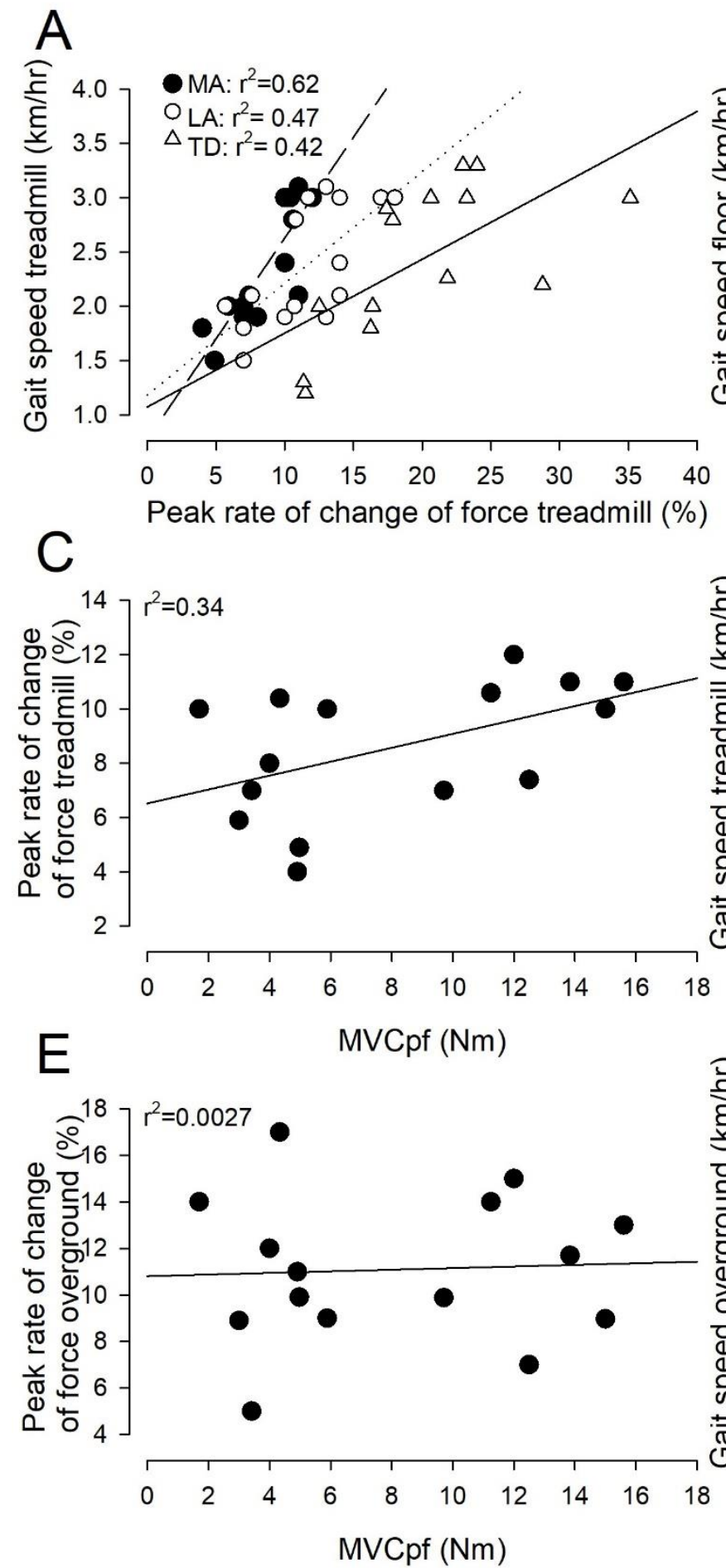

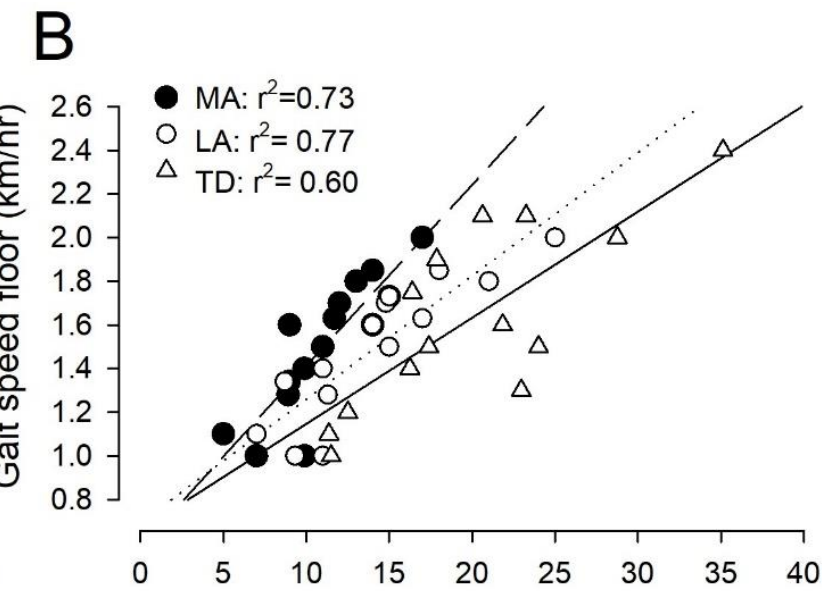

Peak rate of change of force overground ( $\%$
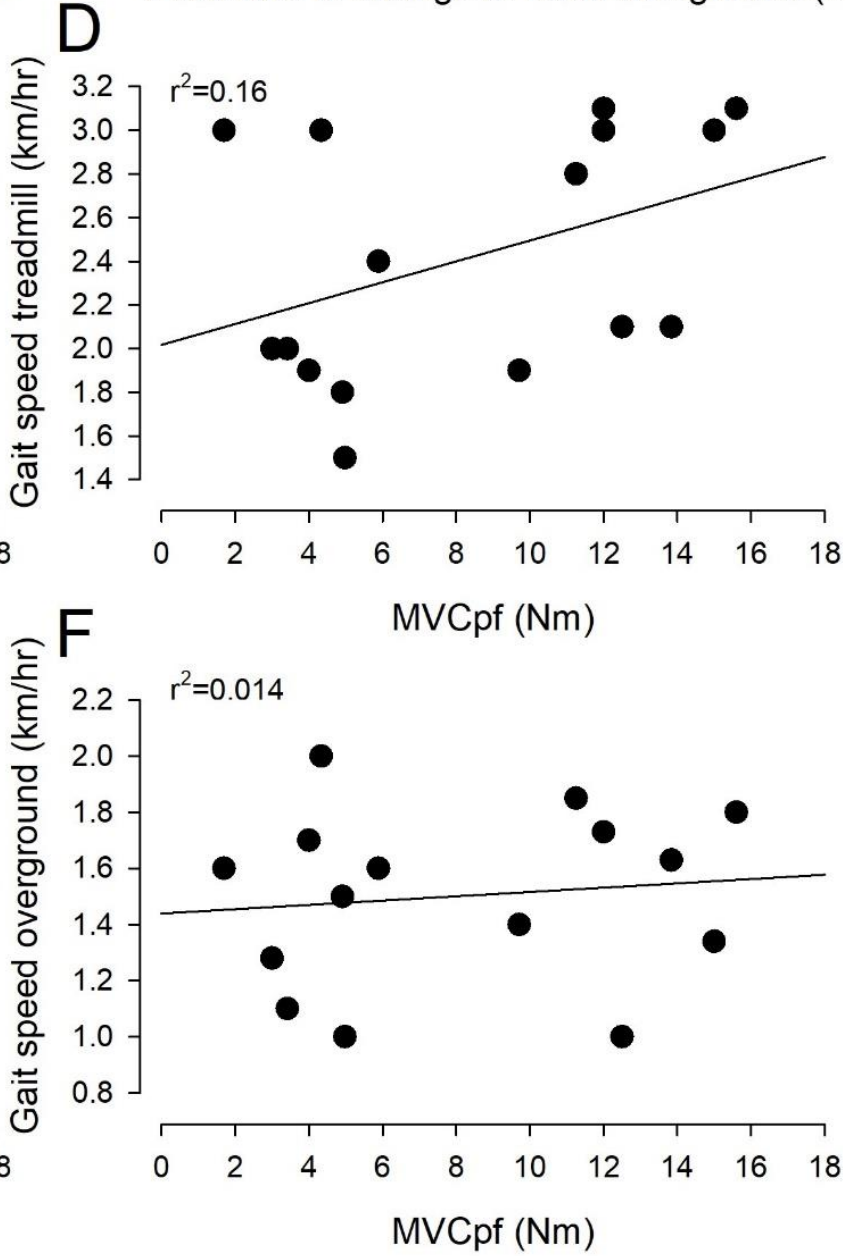

Fig. 2 Relation between MVCpf, push-off force and gait speed during treadmill and overground walking at baseline. A-B show the treadmill (A) and overground (B) gait speed as a function of PJF Closed circles designate measurements from the MA leg and open circles measurements from the LA leg in children with CP. Open triangles designate measurements from TD children. C-F show dPF (C \& E) and gait speed (D \&F) as a function of MVCpf for treadmill (C-D) and overground walking (E-F). In all graphs each symbol represents data from an individual child. Full lines designate regression lines for data from TD children, dashed lines for MA leg in children with CP and dotted lines for the LA leg in children with CP. Correlation coeeficients for the regression lines are given in the upper, left part of the graphs. 

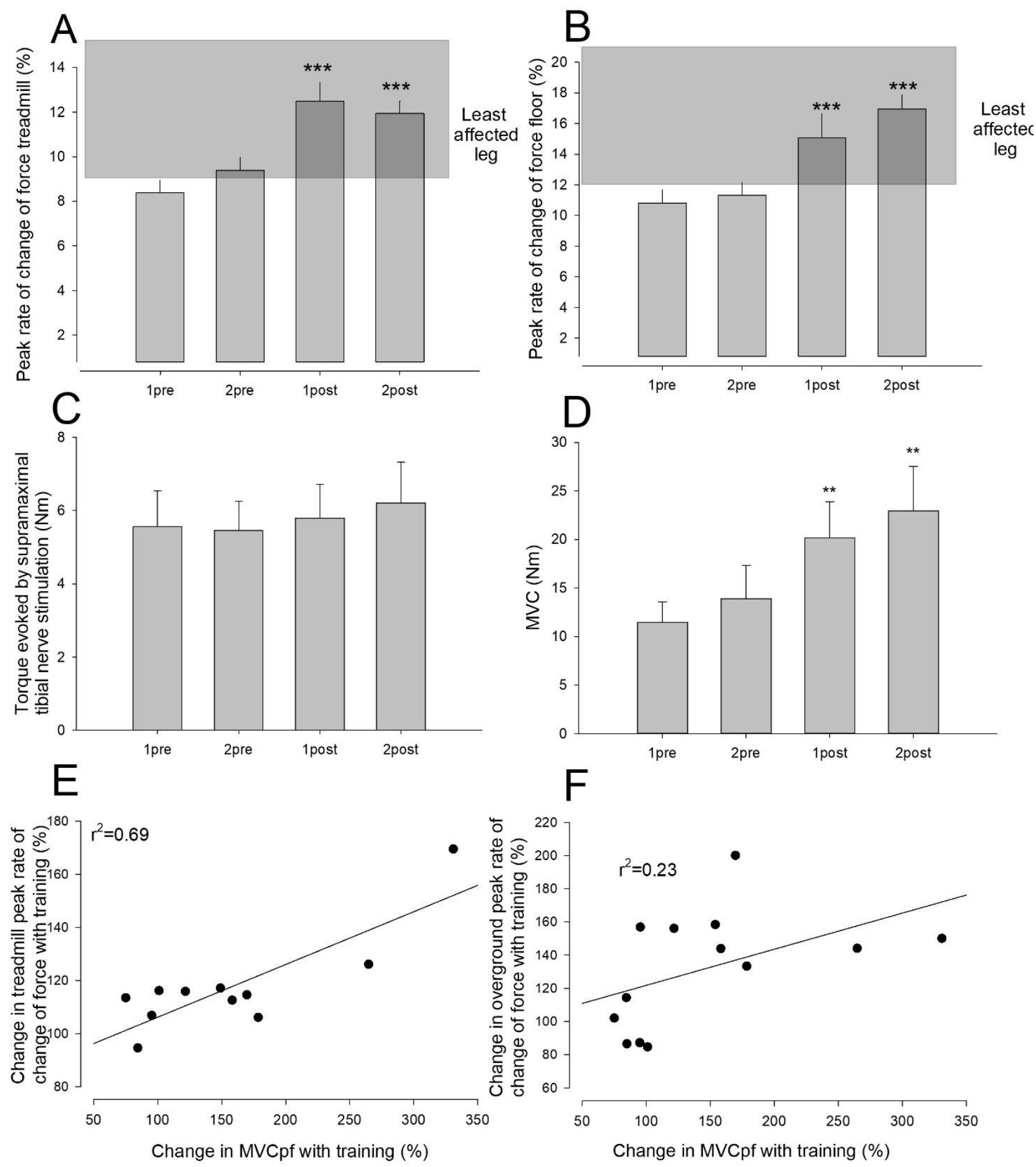

Fig. 3 Effect of 4 weeks of daily treadmill training on push-off force and MVCpf for MA leg. The mean across subjects of dPF during treadmill walking (A), the mean across subjects of dPF during overground walking (B), the average plantarflexor torque evoked by supramaximal nerve stimulation (C) and the average MVCpf (D) was calculated for the MA leg in the children with CP at the first (1pre) and second (2pre) session prior to training as well as the first (1post) and second (2post) session after the training. All data are the mean of the measurements from all children and vertical bars give the standard errors of the mean. Asterisks in A, B and D designate that measurements at 1post and 2post were significantly different from meaurements at $1 \mathrm{pre}\left({ }^{*}=0.05 ;{ }^{* *}=0.01 ; * *=0.001\right)$. In $\mathrm{E}$ and $\mathrm{F}$ the ratio between the measurement at 1 post and 1 pre sessions is given in percent, ratio calculated forthe push-off velocity (E) and the dPF (F) and plotted against the ratio between MVCs measured at 1post and 1pre sessions for each individual child. In A 
and $B$ the shaded grey rectangles give the range of measurements for the LA leg across the four sessions in the children with CP. The full lines designate the regression lines for the data in the graphs. Each symbol represents measurements from one child.

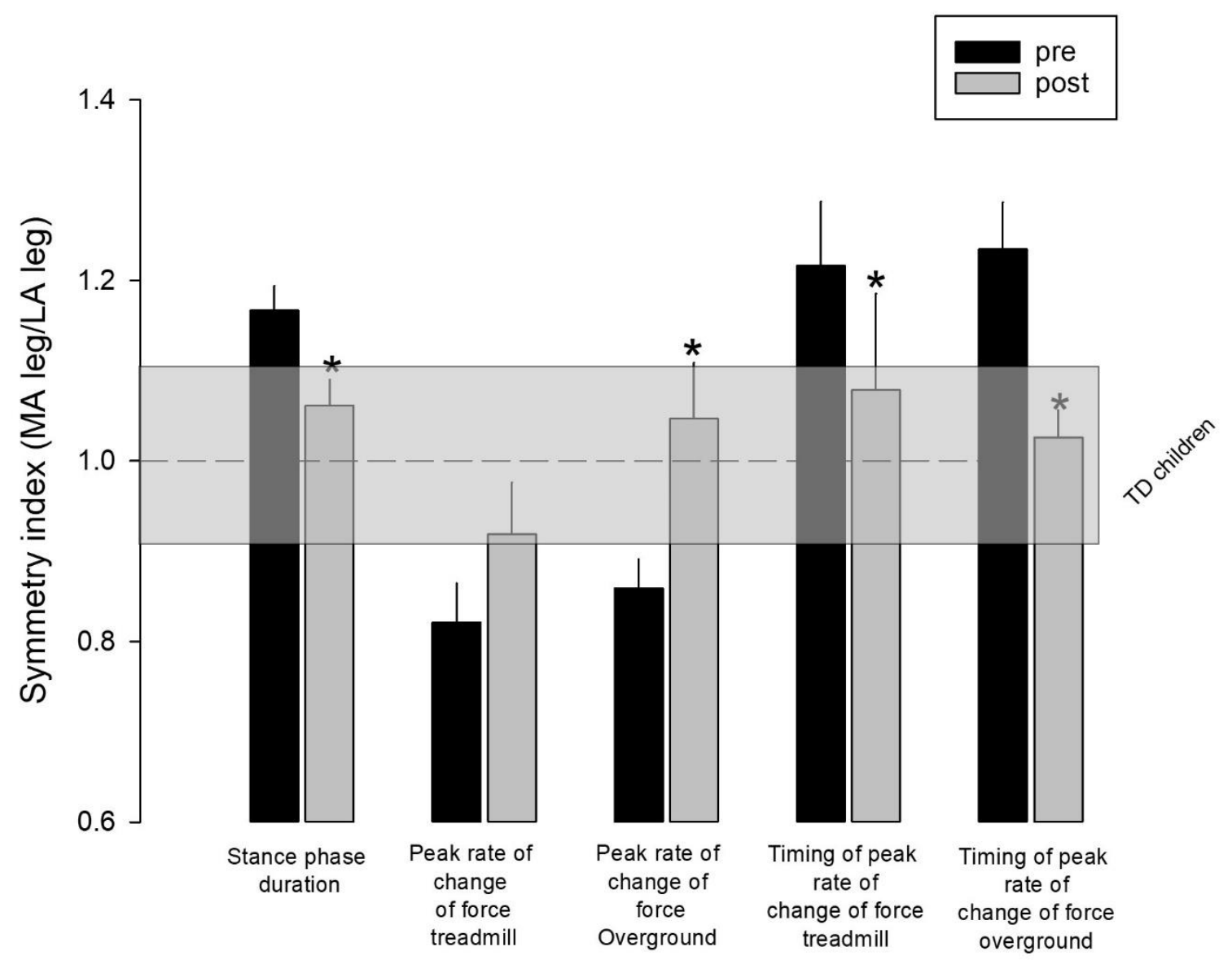

Fig. 4 Changes in symmetry relations between kinematic gait parameters for the least and most affected legs following training

All data show the relation between measurements from the MA limb divided by measurements from the LA leg before ( $1^{\text {st }}$ pre test; black columns) and after training ( $1^{\text {st }}$ post; grey columns)

The dashed horizontal line designates similarity between the two sides. The grey box indicates range of equivalent measures in TD children. Values below 1 designate that the measurement on the MA side was lower than on the LA side. The columns designate from left to right: Stance phase duration (calculated for each leg as interval from ground contact to toes off), the amplitude of the dPF (calculated from the plantar pressure measurements), the amplitude of the peak rate of change of force at push-off during overground walking, the timing of the peak rate of change of force during treadmill walking and finally the timing of the peak rate of change of force during overground walking. 

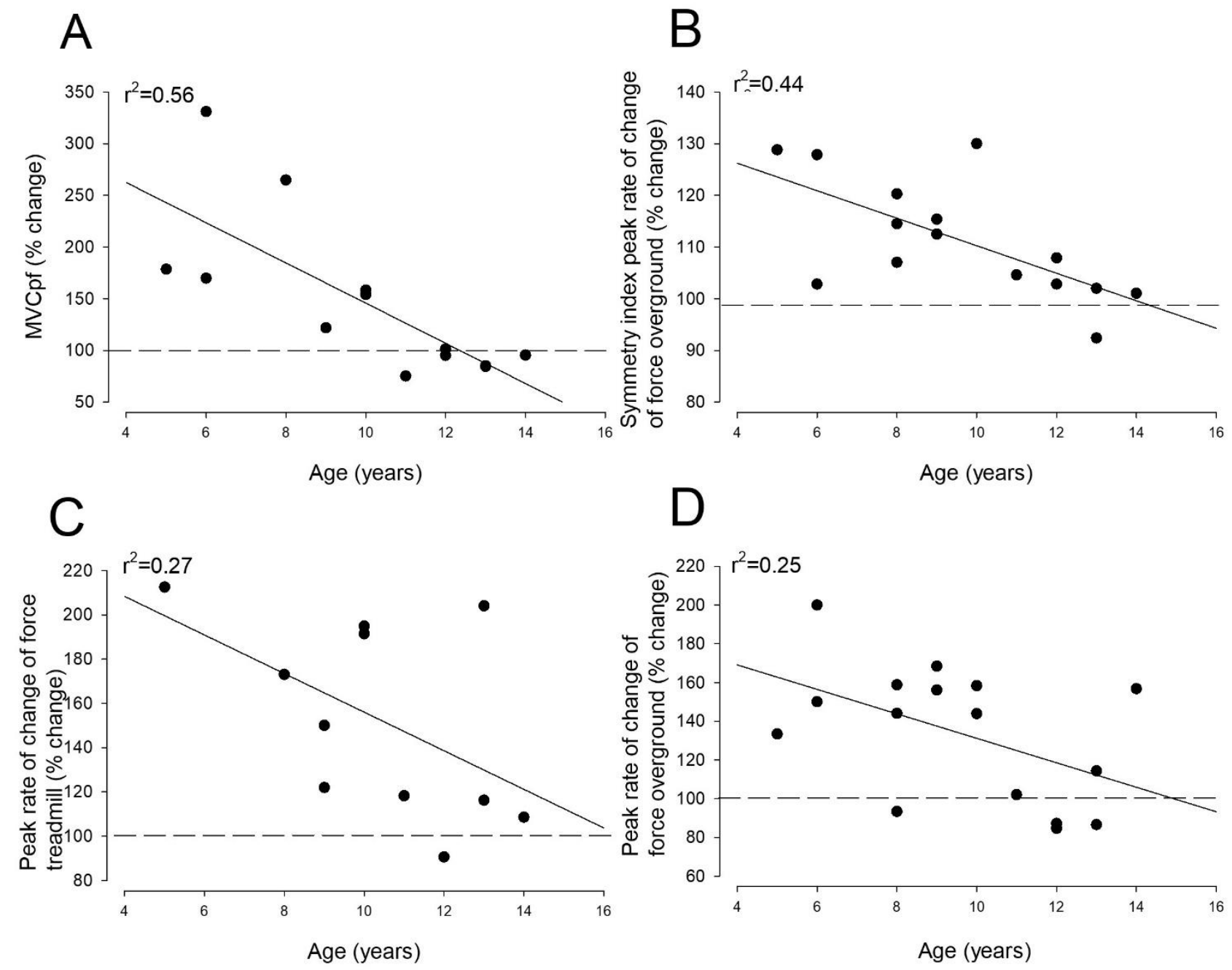

Fig. 5 Age related differences in the effect of training on MVCpf (A), push-off velocity on treadmill (B) push-off jerk force on treadmill (C) and push-off jerk force overground (D).

Each symbol represents measurements from one child the full drawn lines are the regression lines for the data. Correlation coefficients are given in the upper left part of each graph. In all cases the ordinate is the size of the measurement following training ( $1^{\text {st }}$ post training test) as a percentage of the same measurement in the $1^{\text {st }}$ pretest prior to training. The abscissa is the age of the child in years. 